\title{
Anaesthesia for patients with chronic renal disease
}

Patrick H.C. Weir MB BS FRCP(c) Frances F. Chung MB BS FRCP(C)

\author{
CONTENTS \\ Pathophysiology \\ clinical spectrum \\ Preoperative assessment \\ Premedication \\ Anaesthetic considerations \\ Regional anaesthesia \\ General anaesthesia \\ induction agents \\ narcotics \\ neuromuscular blocking agents \\ inhalational agents \\ Perioperative management \\ monitors \\ induction \\ maintenance \\ intraoperative fluid management \\ reversal agents \\ Postoperative care
}

Patients with chronic renal failure have a longer life expectancy than they did two or three decades ago. As a result, anaesthetists now provide care for these patients with increased frequency, for a variety of surgical procedures. Two important factors contributing to the increased life expectancy are the continuing refinements in dialysis and renal transplantation. The ultimate object of dialysis is to maintain chronic renal failure patients until a suitable donor kidney becomes available for transplantation.

The purpose of this article is twofold. We will first review the pathophysiology of chronic renal failure and its effect on the organ systems. The anaesthetic management of patients in chronic renal failure will then be discussed.

\section{Pathophysiology}

Chronic renal failure, regardless of atiology, has one common pathway, resulting in the slow, relent- less destruction of the nephron. The Figure illustrates the four stages through which the renal failure patient passes.

\section{Stage I}

Decreased renal reserve. This initial stage is associated with up to 50-60 per cent loss of nephron mass. Biochemical evidence and symptoms are not usually present.

\section{Stage 2}

Renal insufficiency. With further deterioration in function, mild azotaemia occurs, as the remaining nephrons cannot maintain normal homeostasis. The initial manifestations of hypertension and anaemia appear at this stage. As a result of the impaired ability to elaborate concentrated urine, nocturia is the usual presenting symptom.

\section{Stage 3}

Frank renal failure. With further loss of nephron mass (Glomerula filtration rate (GFR) below 20 per cent of normal), the patient develops overt renal failure. This, in addition to the increased severity of anaemia and hypertension, is characterized by metabolic acidosis, fluid overload and disorcer of other body systems. Hyperkalaemia and hyperphosphotaemia become evident late in this stage.

\section{Stage 4}

Uraemia (Dialysis). Uraemia is simply defined as

\section{Key words}

KIDNEY; failure, chronic, anaesthesia.

From the Department of Anaesthesia, Toronto Western Hospital and University of Toronto.

Address correspondence to: Patrick H.C. Weir, MH, Department of Anaesthesia, Toronto Western Hospital, 399 Bathurst Street, Toronto, Ontario, Canada M5T 2S8. 


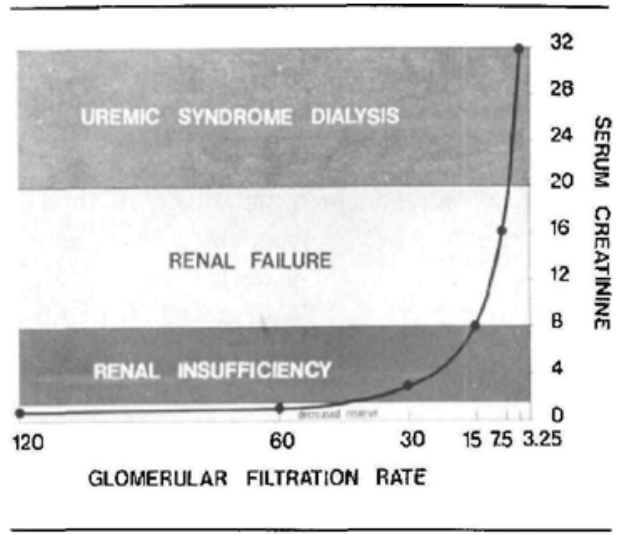

FIGURE Illustration of four stages of loss of renal function Serum creatinine is not markedly increased until reduction in GFR to about one-quarter of normal $(120 \rightarrow 30)$. See lext for details.

excess urea in the blood. As waste products accumulate, cellular metabolism is interfered with and tissue function becomes more and more deranged. The progressive accumulation of hydrogen ions, metabolic waste products and the concomitant fluid overload results in a disordered physiology and ultimate anatomic pathology. Thus, uraemia is a total body disease involving every organ system.'

Haemodialysis, which has usually begun before the more distressing symptoms of uraemia occur, provides both a means of controlling the fluid and electrolyte abnormalities and decreasing the acidosis. ${ }^{2}$

It is of interest to note what occurs at the level of the nephron as renal function declines. When only 30 per cent of the nephrons remain, they must excrete as much as three times more water and solute as would a normal nephron. Hence, an osmotic diuresis occurs in each remaining nephron and this is reflected in the presenting symptoms of polyuria and nocturia. A major consequence of this is a diminution of the ability of the nephron to either dilute or concentrate urine.

The fractional excretion of sodium increases to as high as 50 per cent in patients with a GFR of less than $2 \mathrm{ml} / \mathrm{min}$. There is a potential for a "salt losing defect" with both the solute diuresis and the increased fractional excretion of sodium. ' Concurrent with the increase in fractional excretion of sodium, as nephron mass is lost, there is a decreased flexibility to respond to changes in sodium intake.

These patients will therefore have a tendency to develop oedema and congestive cardiac failure if their intake of water and salt is acutely increased. Conversely, they can become rapidly and severely volume depleted when salt and water intake is decreased. This decreased reserve is of particular importance to the anaesthetist.

Table I summarizes the clinical spectrum of abnormalities seen in chronic renal failure. To assist the systematic approach to the preoperative assess-

TABLE I Clinical spectrum of abnormalities in chronic renal failure

\begin{tabular}{llll}
\hline Cardiovascular & Respiratory & Nervous system & Gastro intestinal \\
\hline Hypertension & "Uraemic lung" & Psychological factors & Mouth ulcers \\
†i Intravascular volume & Infection & Fatigue/lethargy & Parotitis \\
Atherosclerotic heart disease & Airway problems & Muscular iritability & Anorexia, nausea, and vomiting \\
Congestive cardiac failure & & Seizures & Hiccoughs \\
Pericarditis & & Autonomic neuropathy & Delayed gastric cmptying time \\
Chronic pericardial effusion & & Peripheral neuropathy & Gastrointestinal ulceration \\
Cardiomyopathy & Cerebrovascular insufficiency & Constipation \\
Autonomic neuropathy & & Hepatic dysfunction \\
\hline
\end{tabular}

\begin{tabular}{llll}
\hline Haemopoeitic & Endocrine & Metabolic & Immune system \\
\hline Anaemia - normocytic normochronic & Secondary hyperparathyroidism & Acid-base & $\downarrow$ Activity of phagocyres \\
Plasma proteins: $\downarrow$ serum albumin & Adrenal insufficiency & Electrolytes & Immunosuppression \\
Bleeding tendency - platelet dysfunction & Carbohydrate intolerance & & \\
? Hepatitis B carrier & Diabetes mellitus & \\
& Hyperuricemia & & \\
\hline
\end{tabular}


ment of these patients, we shall review each system in turn.

\section{Cardiovascular system}

Two types of hypertension are seen in chronic renal failure. ${ }^{3}$ Patients may be hypertensive as a result of salt and water retention. This hypertension is amenable to treatment with dialysis and sometimes requires additional therapy with digitalis and diuretics.

The second group of patients, fortunately in the minority, have a form of malignant hypertension as a result of high renin output. Their blood pressure is extremely difficult to control and responds poorly to dialysis and antihypertensive medication. Bilateral nephrectomy usually assists blood pressure control.

Longstanding hypertension results in left ventricular hypertrophy. With decreased myocardial contractility and chronic fluid overload, congestive cardiac failure eventually occurs. The anaemia of chronic renal failure further aggravates cardiac failure because of the compensatory increase in cardiac output.

Renal failure patients, especially those undergoing chronic dialysis, are prone to accelerated atherosclerosis. ${ }^{3} \mathrm{~A}$ high percentage of these patients have coronary artery disease.

A painless pericarditis is sometimes present. This was a greater problem when systemic heparinization was used for dialysis, as some patients developed acute pericardial tamponade.

In chronic renal failuse there is the potential for chronic pericardial effusions to develop. Impeded venous return may lead to major haemodynamic instability, particularly during induction.

Uraemic cardiomyopathy occurs in patients with longstanding disease. This will result in a further reduction in contractility and a decreased ejection fraction.

A not uncommon finding in these patients is autonomic neuropathy, which results in a generalized decrease in sympathetic response to hypovolemia, including an abnormal response to the Valsalva manoeuvre.

\section{Respiratory system}

"Uraemic lung" is an entity characterized radiologically by perihilar pulmonary venous congestion, giving a typical "butterfly wing" type of distribution.
As a result of immunosuppresion, chronic renal failurc patients are prone to pulmonary infection. General anaesthesia and instrumentation of the airway may well result in an exacerbation of preexisting infection.

Thorough assessment of the airway in chronic renal failure patients is important. In our clinical experience renal failure patients present greater intubation problems than do the general population.

\section{Nervous system}

Early symptoms of uraemia include neuromuscular irritability, fatigue, lethargy and mild behavioural changes.

Peripheral neuropathy becomes clinically evident in many uraemic patients and its early appearance is generally considered a firm indication to initiate dialysis or transplantation. ${ }^{2}$ The common peroneal and median nerves are the ones most frequently involved. The "restless leg syndrome" is a common presenting symptom. Due to the potential medical-legal implications, the anaesthetist may wish to avoid the use of regional anaesthesia affecting the involved limb.

Patients with terminal uraemia are prone 10 seizures and eventually will develop uraemic encephalopathy. In chronic renal failure, seizure disorders may also present in two other circumstances.

Patients with malignant hypertension are prone to develop cerebral oedema, which may precipitate convulsions and possibly coma.

Secondly, patients on chronic haemodialysis may develop "dialysis dementia," and the "disequilibrium syndrome," two types of neurological disturbances resulting in seizure disorders. ${ }^{2,3}$

\section{Gastrointestinal system}

Nausea, vomiting and recurring episodes of hiccoughs are some of the more distressing symptoms incurred by those with renal failure. The symptoms can complicate the anaesthetic induction and make these patients prone to aspiration of gastric contents. Renal failure patients have a delayed gastric emptying time, to approximately 300 minutes. This has been shown to almost double when the patient is undergoing haemodialysis. ${ }^{4}$

Hyperacidity and an increase in gastric volume are also common findings in chronic renal failure. ${ }^{3}$ We therefore regard all these patients as potentially 
having a "full stomach." Pre-oxygenation and a rapid scquence induction are advised.

The most common hepatic abnormalities include hepatitis (both viral and drug related), chronic hepatic venous congestion and haemosiderosis. Viral hepatitis, although not a complication of wraemia per se, is a common problem encountered in chronic dialysis patients. Type B hepatitis is most common and antigenaemia usually persists long after the clinical features of hepatitis have resolved. Hepatic dysfunction secondary to chronic hepatic venous congestion is seen in about five to ten per cent of patients with chronic renal failure. ${ }^{5}$

\section{Haemopoietic}

A normocytic, normochromic anaemia invariably occurs in patients with chronic renal failure. The haemoglobin levels rangc between $30-90 \mathrm{~g} / \mathrm{L}$, coexistent with haemotocrit levels of $15-25$ per cent.

The aetiology of this anaemia is multifactorial. ${ }^{1,3}$ The main cause, however, is a decrease in the production of renal erthropoietin. Other contributing factors include: (1) decreased half life of the red blood cells, (2) increased red cell membrane fragility, (3) haemolysis, and (4) decreased bone marrow production.

Deficiencies of iron, folic acid, vitamins $B_{12}$ and $\mathrm{B}_{6}$ play a lesser role, concomitant with chronic loss of red blood cells due to gastrointestinal ulceration.

The greatest hazard of anaemia is the decrease in the oxygen-carrying capacity, usually to that of half of normal. The major compensatory factor for this decreased reserve is an increase in cardiac output. In addition, the release of oxygen from haemoglobin to the tissues is facilitated by a shift to the right of the oxyhaemoglobin dissociation curve. This shift is due to metabolic acidosis and an increased concentration of 2,3-diphosphoglycerate.

Patients with chronic renal failure tolerate general anaesthesia quite well and preoperative transfusion is not usually indicated. Previously, transfusions for potential kidney transplant candidates were avoided, to minimize sensitization to histocompatability antigens. More recently however, opinions have changed dramatically on this matter.

Opelz, Terasaki and others have confirmed a higher degree of graft survival in renal transplantation patients who were transfused prior to surgery. ${ }^{6-8}$ Preoperative transfusion would also be indicated for those renal failure patients presenting for major surgery. ${ }^{9}$ Thawed frozen erythrocytes that have been washed in saline should be used.

Platelet dysfunction in uraemia is characterized by a prolonged bleeding time, a decrease in platelet adhesiveness and abnormal prothrombin consumption. ${ }^{10}$ It has been suggested that an accumulation of guadininosuccinic acid, phenol and phelolic acid cause a decline in factor three availability. ${ }^{10}$ This, in turn, results in an inhibition of secondary platelet aggregation. Adequate haemodialysis effectively eliminates the offending compounds and ustally restores adequate platelet function. ${ }^{11}$

"Rebound heparinization" from haemodialysis may aggravate the bleeding tendency of uraemia. ${ }^{12}$ The use of "regional heparinization" techniques in the dialysis machine seems to have solved this problem.

It should be noted that those patients with hepatic dysfunction may have a reduction of intrinsic clotting factors produced by the liver, making things even more complicated. Because of this bleeding tendency, a full coagulation screen shouid be done prior to major surgery.

\section{Endocrine system}

Uraemic osteodystrophy is a broad term that encompasses a number of distinct skeletal abnormalities including osteomalacia, osteosclerosis and osteitis fibrosa cystica, the latter being described as the bone changes seen with secondary hyperparathyroidism.

As renal function declines, so does the excretion of phosphate. The resultant hyperphosphataemia, along with the decreased gastrointestinal absorption of calcium, results in hyperactivity of the parathyroid gland which is trying to maintain normal serum calcium levels. Metastatic calcification occurs in the soft tissues when the calcium-phosphate product exceeds $60 .^{3}$ The long-term result is generalized bone decalcification which makes these patients very prone to spontaneous fractures. Thus, great care in positioning should be taken by operating room personnel.

Corticosteroid therapy is relatively common in this population group. As a result, these patients will be at risk during surgery because of adrenal insufficiency. Perioperative steroid coverage will be required.

The ability to metabolize an exogenous glucose load is impaired in most patients with chronic renal 
failure. This "glucose intolerance" is manifested by an abnormal glucose tolerance test and tends to be somewhat resistant to treatment with insulin. Diabetes mellitus, with its consequent end organ damage, often is present in chronic renal failure.

\section{Acid base - electrolytes}

A mild metabolic acidosis becomes evident when the GFR drops to below 25 per cent of normal. This acidosis is characterized by a reduction in serum $\mathrm{HCO}_{3}$, a compensatory fall in $\mathrm{PCO}_{2}$ and a slight decrease in pH. Initially, the anion gap is normal, but as renal function deteriorates there will be an increase in the anion gap due to the retention of sulphates and phosphates.

Hyponatraemia is usually mild, reflecting the dilutional effect of increased total body water. Diuretics tend to worsen the hyponatraemia.

The most serious electrolyte abnormality in patients with chronic renal disease is hyperkalaemia. Hyperkalaemia is rarely responsible for clinical symptoms unless the GFR is below $5 \mathrm{ml} / \mathrm{min}$ or an endogenous (haemolysis, trauma, infection) or exogenous (stored blood) potassium load is administered.

It is generally recommended that elective surgery not be performed unless the serum $\mathrm{K}^{+}$is less than $5.5 \mathrm{mEg} / \mathrm{L}^{13}$ If surgery cannot be delayed, the serum $\mathrm{K}^{+}$concentration can be lowered by the administration of glucosề-insulin infusions along with hyperventilation. Intravenous calcium is effective in restoring normal cardiac conduction. Sodium bicarbonate is indicated if a metabolic acidosis accompanies the hyperkalaemia. The implications of succinylcholine and hyperkalaemia will be discussed later.

Hypermagnesemia is a problem only in patients who abuse magnesium-containing antacids. The neuromuscular effects of hypermagnesemia, especially the potentiation of neuromuscular blocking drugs, are well documented. ${ }^{14}$

\section{Preoperative assessment}

In the preoperative assessment of patients with renal disease, a thorough history and physical examination is necessary. The anaesthetist should be alert for signs and symptoms encompassing the clinical spectrum of abnormalities seen in chronic renal failure. Particular attention should be paid to the intravascular volume status. The dialysis pattern
TABLE II Medications commonly taken by patients with chronic tenal failure

Digitalis

Beta adrenergic bleckers

Antihypertensive agents

Diuretics

Antiarthythmic agents

Nitrates

Cortico-steroids

Immunosuppressive agents

Other (e.g., anticonvulsants, antibiotics)

(e.g., frequency of dialysis, dry weight, etc.) and the time of the last dialysis prior to surgery should be noted

Table II lists the common medications taken by patients with chronic renal disease. The majority have potential influence on anaesthetic management.

Digitalis is excreted by the kidney and is usually administered at a lower dosage in uraemic patients. Signs and symptoms of toxicity should be carefully monitored.

Many patients, particularly those with high renin output hypertension, are receiving a combination of beta adrenergic blockers and other antihypertensive agents. These agents should be continued until the time of surgery. The impact of these drugs usually results in a reduction of cardiac contactility and a reduced vascular reactivity.

Diuretics can result in hypovolemia and hyponatraemia. In patients with early renal failure, it is not uncommon to see diuretic induced hypokalaemia.

Antiarrhythmic and anti-anginal agents are often taken by patients with ischaemic heart disease. The anaesthetic implications of calcium channel blocking drugs is well documented. ${ }^{15,16}$ Corticosteroids and immunosuppressive agents are usually administered to patients who have undergone previous renal transplantation.

The preoperative assessment should include examination of all pertinent laboratory data. Serum clectrolytes, blood urea nitrogen, serum creatinine, acid base status, haemoglobin, haemotocrit, bleeding time, prothrombin time and partial thromboplastin time are the most important laboratory results. Most of the blood work will be done shortly after dialysis. If there is a time lapse of more than six hours between dialysis and surgery, the electro- 
lytes should be repeated, since the serum $\mathrm{K}^{+}$can rise dramatically in some patients. A recent chest $X$-ray and EKG should also be obtained.

\section{Premedication}

The objective of premedication is to achieve adequate sedation without cardiovascular or respiratory depression. It should be remembered that not only do these patients metabolize drugs abnormally, but they also have a limited capacity to excrete certain drugs. Consideration should also be given to the type of surgery planned as well as the clinical condition of the patient.

At one end of the spectrum is the young. somewhat robust and nervous patient who will probably benefit from a full dose of narcotic premedication.

Those with terminal uraemia are at the other end of the spectrum. They are very sensitive to all psychotrophic drugs. We do not administer any narcotic or sedative medications to these patients until they are under the direct surveillance of an anaesthetist.

The newer and shorter-acting benzodiazepines such as oxazepam and tempazepam are recommended in renal failure. ${ }^{17}$ Anticholinergics are not usually recommended as part of the premedication routine. Renal failure patients have dry, friable mucous membranes and the administration of this type of agent can make the patient very uncomfortable. If there is a specific indication for a vagolytic agent, glycopyrrolate can be administered intravenously at the time of induction.

Due to gastric hyperacidity it would also seem prudent to use a non-particulatc antacid along with an $\mathrm{H}_{2}$ receptor antagonist. Oral cimetidine $(400 \mathrm{mg}$ ) or ranitidine $(150 \mathrm{mg}$ ) given four hours preoperatively are suggested. ${ }^{18,19}$

Insulin infusions and corticosteroid coverage, if indicated, are also part of the preoperative preparation of these patients.

\section{Anaesthetic considerations}

Table III outlines the special anaesthetic considerations in chronic renal failure.

Most patients are aware of the chronic nature of their illness and its serious prognosis. They have probably undergone surgery on numerous occasions. Understandably, they have a wide range of psychological problems, ranging from mild depres-
TABLE III Anaestheric considerations

\begin{tabular}{l} 
Factors-Surgical \\
Anaesthetic \\
$\mathrm{O}_{2}$ carrying capacity \\
Intravascular volume status \\
Coronary artery disease \\
Hyperten sion \\
Immunosuppression (aseptic technique) \\
Acid base - electrolytes ( $\mathrm{K}^{+}$) \\
Potential full stomach \\
Drug excretion \\
Minor \\
Bleeding tendency \\
Venous access \\
Bone decalcification \\
? Hepatitis B antigen carrier \\
Protection of shunts/fistulae \\
Plasma cholinesterase level \\
\hline
\end{tabular}

sion to frank psychotic behaviour. The anaesthetist should be prepared to spend more time than usual when visiting these patients preoperatively. Establishment of a good rapport will probably be beneficial as you are about to make your sixth attempt at establishing an intravenous line!

Surgical factors will depend on the nature and the site of the surgery. For example, the factors involved in renal transplantation will include: (1) as the surgery is extra-peritoneal, only moderate relaxation is required, (2) there is a potential for severe haemorrhage, and (3) intraoperative administration of methylpredniodone (Solumedrol) and diuretics may be required.

Because of the decrease in oxygen carrying capacity, an $\mathrm{FIO}_{2}$ of 0.4 or 0.5 is appropriate. If major surgery is contemplated, preoperative transfusion may be indicated. With respect to the intravascular volume, it is probably wise to err on the "full" side. Tasker et al. have shown that the prophylactic administration of saline preoperatively in patients with chronic renal failure was effective in preventing further deterioration in renal function. ${ }^{9}$ Carlier $e t$ al. found that maximal hydration during anaesthesia improved the early function of human renal transplants. ${ }^{20}$

Patients with coronary artery disease or hypertension will require direct arterial pressure monitoring. Careful control of the rate-pressure product on 
induction and during maintenance of anaesthesia is required. Those patients with poor left ventricular function or recent myocardial infarction $(<$ three months) should have a pulmonary artery catheter inserted prior to anaesthesia. A moderate to high dose fentanyl-oxygen technique (fentanyl 50 $100 \mu \mathrm{g} \cdot \mathrm{kg}^{-1}$ ) is recommended for patients in this category.

Strict aseptic techniques are necessary when inserting central venous and arterial lines in those patients who are immunosuppressed.

Drugs excreted entirely by the kidney should be avoided. This will be further discussed in the section on general anaesthesia.

Venous access is usually difficult. It is preferable not to use the veins of the forearm, as they may be needed for the creation of a future arterio-venous fistula.

If the patient is a proven carricr of the hcpatitis $B$ virus, full precautions should be taken by all operating room personnel.

It is the responsibility of the anaesthetist to ensure that all shunts or fistulae are adequately protected. The involved arms should be padded with a cotton web roll, and preferably be placed on an am board. The patency of the shunt can be monitored continuously by using a doppler probe.

\section{Regional anaesthesia}

Regional anaesthesia in patients with chronic renal failure is a logical choice, as it avoids the effects of muscle relaxants, narcotics and potent volatile anaesthetics. Intubation is not required, thereby decreasing the risk of iatrogenic pulmonary infection from anaesthetic equipment. Also, the risk of aspirating gastric contents is avoided when these anaesthetic techniques are employed. Minor nerve blocks and plexus anaesthesia are indicated for procedures involving the extremities (e.g., placement of an arterio-venous shunt or fistula).

Table IV lists potential limitations in the consideration of regional anaesthesia.

The bleeding tendency of uraemia could theoretically be a problem with spinal or epidural anaesthesia, due to the risk of haematoma formation. However, if the patient is on an adequate dialysis programme, the primary platelet dysfunction is reversed. ${ }^{10,11}$ If both the coagulation screen and bleeding time are normal, there should be no objection to the use of spinal or epidural techniques.

Epidural anaesthesia was advocated for renal
TABLE IV Regional anaesthesia

\section{Potential limitations}

Bleeding tendency

Sympathetic blockade

Patient acceptance

? Inadequate duration

Uraemic neuropathy

? Epinephrine usage

Metabolic acidosis ( $\downarrow$ C.N.S. threshold)

transplantation by Wyant (1967), ${ }^{21}$ while Vandam et al. $(1962)^{22}$ used continuous spinal analgesia. More recently, Linke reports on 64 patients who were managed successfully using spinal anaesthesia for this procedure. ${ }^{23}$ There have been no reports of epidural haematoma formation.

Sympathetic blockade is an important consideration when utilizing epidural or spinal anaesthesia. A high level of blockade can result in a significant drop in blood pressure and GFR. Careful preoperative fluid loading will help offset the degree of hypotension. It should be remembered that patients with autonomic neuropathy will not be able to adequately compensate for a fall in blood pressure as would a nomal patient. Spinal and epidural anaesthesia should be avoided in patients with severe hypertension.

Regional techniques may be unsatisfactory for prolonged procedures, due to both inadequate duration and poor patient acceptance. Bromage reported a 38 per cent reduction in the duration of brachial plexus block in patients with renal disease. ${ }^{24} \mathrm{He}$ attributed this to a faster tissue washout of the local anaesthetic, secondary to the increased cardiac output. However, brachial plexus block is still considered the anaesthetic of choice for the creation of an arterio-venous fistula.

The use of regional anaesthesia in patients with uraemic neuropathy is controversial. Most anaesthetists, as previously discussed, will agree that its use is contraindicated, although some authors differ. ${ }^{23,25}$

The use of epinephrine to prolong local anaesthetic action should be considered carefully in the acidotic, hyperkalaemic patient, due to the risk of cardiac arrhythmias.

As acidosis decreases the central nervous system threshold to the toxic effects of local anaesthesia, the total dose of anaesthetic should be decreased by approximately 25 per cent in the acidotic patient. Also, a recent report of bupivacaine cardiotoxicity 
in a patient with renal failure suggests that the total anaesthetic dosage should be reduced. ${ }^{27}$

\section{General anaesthesia}

Induction agents

\section{SODIUM THIOPENTONE}

Thiopentone is generally considered the induction agent of choice for patients with chronic renal failure. It should be given slowly and titrated carefully, according to the physical status of the individual patient. Ghoneim and Pandya found that thiopentone is 50 per cent unbound in uraemic patients, compared to 28 per cent in control patients. ${ }^{28}$ The increase in the non-ionized, or active portion of the drug is due to several factors.

Thiopentone binds to serum albumin. Albumin levels are decreased in uraemia. It is also postulated that albumin-bound thiopentone is displaced by nitrogenous end products. ${ }^{28}$ Acidosis may also increase the amount of the non-ionized form, since the $\mathrm{pK}$ of thiopentone (7.6) is close to the physiological pH. There is evidence that the blood-brain barrier may not bc intact in the presence of uraemia, making these patients particularly sensitive to lipid soluble agents such as thiopentone. ${ }^{29}$

\section{KETAMINE}

Ketamine is metabolized by the liver and excreted by the kidney. Because of its sympathomimetic properties, it should not be used in patients with hypertension and coronary artery disease.

\section{DROPERIDOL}

Droperidol, in combination with fentanyl, causes a small decrease in GFR, effective renal blood flow and urine output. ${ }^{30}$ Neurolept-analgesia has been recommended as a safe technique in patients with renal failure. ${ }^{31,32}$

Moderate to high dose fentanyl techniques are usually reserved for the high-risk patient. Highdose fentanyl attenuates the stress response to surgery, resulting in reduced serum catecholamine levels and increased free water clearance. ${ }^{33} \mathrm{Oxy}$ gen alone can then be used, thus avoiding the myocardial depressant effcts of nitrous oxide.

\section{Narcotics}

Morphine and meperidine, until recently, were thought to cause a decrease in urine output by stimulation of antidiuretic hormone (ADH). It is now evident that the ADH release is secondary to surgical stimulation. ${ }^{34}$ Although most of the narcotics undergo hepatic elimination, urine output may influence their duration of action. ${ }^{35}$ The dose of narcotics should be reduced in advanced renal failure.

With repeated doses of meperidine, normeperidine (an active metabolite of meperidine) accumulates in some patients with renal failure. This accumulation of normeperidine may result in irritability, twitching or seizures. ${ }^{36}$ Fentanyl would appear to be the narcotic of choice in chronic renal failure, as it causes minimal haemodynamic changes and has a short half life when administered in the low dose range.

\section{Neuromuscular blocking agents}

\section{SUCCINYLCHOLINE}

Succinylcholine is commonly used for rapid sequence induction and is a useful agent in chronic renal failure because of the delay in gastric emptying time. Intravenous succinylcholine causes the release of potassium from skeletal muscle during the process of depolarization. A rise in serum potassium of between 0.5 to $0.7 \mathrm{mEg} / \mathrm{L}$ is found in both normal patients and those with renal failure. ${ }^{37}$ Precurarization does not attenuate this rise in serum $\mathrm{K}^{+}$.

It is generally considered safe to use succinylcholine in renal failure if the serum potassium is less than $5.5 \mathrm{mEq} / \mathrm{L}^{38}$ Succinylcholine should be avoided in patients with advanced uraemic neuropathy, since an exaggerated hyperkalaemic response can result. ${ }^{39}$

There have been controversial reports regarding serum cholinesterase activity in renal failure. In the early days of dialysis, the cellophane type of membrane was used. It is known that this type of membrane did lower the serum cholinesterase. ${ }^{21}$ The newer membranes do not cause this problem. Ryan found that cholinesterase activity was not reduced in chronic renal failure. ${ }^{40}$ Clinically prolonged apnoea following succinylcholine in renal failure does not seem to be a problem.

\section{NONDEPOLARIZING MUSCLE RELAXANTS}

Many patients with chronic renal failure have decreased muscle mass and require a reduction in the total dosage of nondepolarizing agents. Also, in 
the absence of renal blood flow, the distribution volume of non-depolarizing muscle relaxants is reduced, resulting in higher blood concentrations. ${ }^{41}$

Potentiation of neuromuscular blockade may occur with respiratory and metabolic acidosis. Other potentiating factors in renal failure include hypokalaemia, hypocalcaemia, hypermagnesaemia, certain antibiotics (e.g., aminoglycosides) furosemide and mannitol. ${ }^{14,42,43}$ Therefore, it is important to give the minimal dosage that is required and to monitor neuromuscular activity with a peripheral nerve stimulator.

\section{D-TUBOCURARINE}

d-Tubocurarine is the preferred non-depolarizing muscle relaxant in renal dysfunction as there is no increased sensitivity in renal failure. Cohen, in anephric dogs, demonstrated that within a few hours, the liver was able to excrete up to 40 per cent of a given dose of curare. ${ }^{44}$ The disadvantages of curare are ganglionic blockade and peripheral vasodilatation, both of which may result in hypotension.

\section{PANCURONIUM}

Pancuronium is more dependent upon renal excretion and less able to be excreted by the biliary tract. ${ }^{41}$ There is some hepatic transformation to less active or inactive metabolites. Prolonged paralysis has been reported following the administration of pancuronium. Its advantage is cardiovascular stability.

\section{METOCURINE}

There is much less biliary excretion than with d-tubocurarine and no metabolites have been demonstrated. It is not sujtable for chronic renal failure patients.

\section{GALLAMINE}

Its use is contraindicated, because it depends on renal excretion.

\section{VECURONIUM AND ATRACURIUM}

Vecuronium, the monoquatemary homologue of pancuronium, is a new competitive neuromuscular blocking agent without significant cardiovascular effects. Animal experiments have shown significant biliary excretion. Its use in renal failure patients is encouraging. ${ }^{45}$

Atracurium, another new agent, has a short duration of action because it is broken down by both Hoffman elimination and non-specific esterases. Preliminary evidence shows that it is ideal for patients with chronic renal failure. ${ }^{46,47}$

\section{Inhalational agents}

The advantage of inhalational agents lies in their rapid elimination from the body, independent of renal function. They are useful for controlling intraoperative hypertension and in reducing the total dose of muscle relaxant. However, they can cause excessive depression of myocardial contractility which results in a fall in cardiac output. The fall in blood pressure will lead to a reduction of both renal blood flow and glomerular filtration rate.

There is also increasing concern over fluorideinduced renal toxicity. The degree of renal tubular damage is proportional to the peak fluoride ion levels. These levels depend on the degree of metabolic degradation of the fluorinated agents (methyoxyflurane $\gg$ enflurane $>$ isoflurane in a ratio of 10:2:1).

\section{METHOXYFLURANE}

Methoxyflurane is containdicated in patients with renal disease due to the nephrotoxic effects of high serum fluoride ion levels occurring as a result of metabolism. ${ }^{48}$

\section{ENFLURANE}

Enflurane is metabolized to a lesser extent than methoxyflurane. ${ }^{49}$ The inorganic fluoride levels seldom exceed $20 \mu \mathrm{m} / 1$, which is below the considered nephrotoxic threshold of $50 \mu \mathrm{m} / \mathrm{l}$. Mazze recently has shown enflurane does not cause further deterioration of renal function in surgical patients with mild to moderate renal insufficiency. ${ }^{30}$ However, there are three case reports of renal failure associated with high fluoride levels after enflurane anaesthesia. ${ }^{51-53}$ It has been postulated that the threshold for fluoride-induced nephrotoxicity may be lower in diseased or transplanted kidneys. ${ }^{52}$ Caution should be exercised in the use of enflurane in patients with renal dysfunction, especially if other exacerbating factors such as prolonged anaesthesia, obesity and enzyme induction are present.

\section{ISOFLURANE}

Isoflurane undergoes less hepatic biotransformation than its isomer, enflurane. ${ }^{54}$ The resultant 
inorganic fluoride levels are very low. This property, along with its low arrhythmogenicity, make it an ideal agent for patients with renal failure.

\section{HALOTHANE}

Halothane causes minimal elevation in serum fluoride levels. Deep halothane anaesthesia may reduce renal blood flow by 30 per cent. ${ }^{55}$ It can be safely used if cardiovascular stability is maintained.

\section{Perioperative management}

When the patient arrives in the operating room, the appropriate monitors should be applied (see Table V). Preoxygenation for five minutes is recommended to faciltate denitrogenation. During this time, precurarization and additional sedation, if required, are given. A sleep dose of thiopentone is carefully administered, followed by succinylcholine. The trachea is intubated while cricoid pressure is applied.

Maintenance of the anaesthetic state is achieved using either halothane or isofurane and nitrous oxide $\left(\mathrm{F}_{1} \mathrm{O}_{2} \mathrm{O} .4\right.$ to 0.5$)$. Fentanyl is the narcotic of choice. If the blood pressure is normal following induction, small increments of tubocurarine are given until the desired effect is reached. If the blood pressure has fallen, pancuronium should be used. Atracurium and vecuronium, soon to be available, will possibly become the agents of choice in the presence of renal failure.

Meticulous attention should be paid to ventilation, aiming at normocapnia. The deleterious effects of hypo or hyperventilation are more pronounced in these patients.

The treatment of intraoperative hypotension should be directed at the cause. Vasopressors should be avoided due to their adverse effects on rcnal blood flow. Treatment should include volume expansion, the Trendelenberg position and if necessary, the use of dopamine in the low dose range.

Intraoperative fluid administration should be restricted and volumes used should be aimed at replacing specific losses.

The following regimen is suggested:

(1) replacement of insensible water loss with five per cent dextrose ( $500 \mathrm{mls} / \mathrm{day}$ in the adult),

(2) replacement of urine output with 0.45 per cent saline,

(3) replacement of blood loss with fresh, salinewashed packed red cells,
TABLE V General anaesthesia for renal failure patients

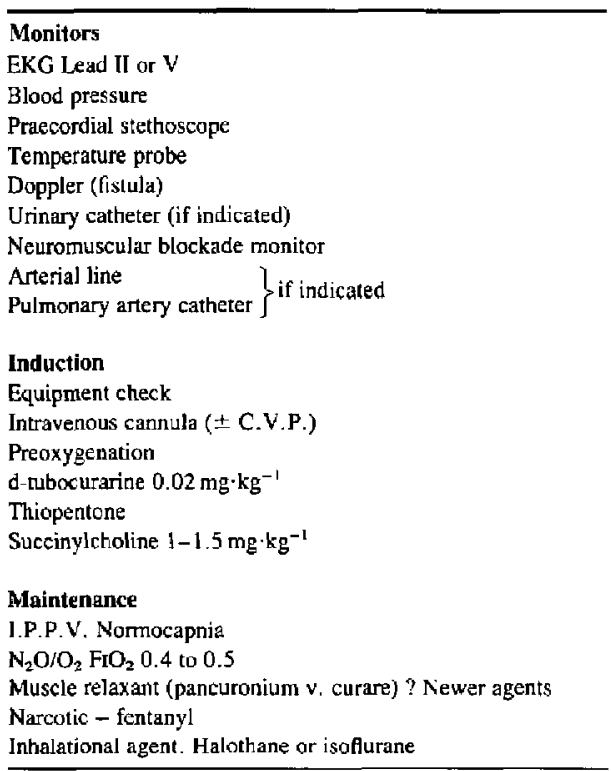

(4) replacement of "third space" loss with normal saline, alternating with isotonic plasma protein solutions at the rate of 2 to $5 \mathrm{~m} / \mathrm{s} / \mathrm{kg} / \mathrm{hr}$, depending on the extent, location and duration of tissue trauma. Potassium containing solutions should be avoided.

Modifications of this regimen must be made when monitors (blood pressure, central venous pressure, capillary wedge pressure and urine output) indicate that fluid therapy is either inadequate or excessive.

Adequate reversal of muscle relaxants can usually be achieved with neostigmine. The kidney excretes approximately 50 per cent of neostigmine. In the presence of renal dysfunction, the serum half life of neostigmine is prolonged. $\$ 6$ However, we prefer to use pyridostigmine as it has a longer half life than neostigmine ${ }^{57}$ It is probably more suitable in renal failure, due to the possibility of "recurarization." 58

Glycopyrrolate has a longer half life than atropine and is the preferred anticholinergic. It is more suitable for patients with hypertension and coronary artery disease as it has a lesser antimuscurinic effect on the heart. 


\section{Postoperative care}

Care of the post-surgical patient should not end in the recovery room. Postoperative care should include maintenance of normovolemia, stable cardiovascular parameters and adequate urine flow.

Particular attention should be paid to the adequacy of ventilation. The potential for "recurarization" in these patients is high because of the existence of potentiating factors. If there is a weak hand grip, unsustained head lift, or any other factors not meeting the criteria set by Miller, the patient should be electively ventilated until full reversal is present. ${ }^{59}$ Oxygen therapy in the postoperative period should be continued for four to six hours.

Frequency measurements of serum electrolytes, particularly potassium, is necessary.

Ideally, the surgeon, anaesthetist and nephrologist should interact to provide a team approach in the care of these patients following surgery.

\section{Acknowledgments}

We would like to thank Dr. William Jones and Dr. Ronald Crago for their help in reviewing this article. Also special thanks to Mrs. Isabel Bell for her secretarial assistance and Mrs. Dianne Weir for proof-reading the article.

\section{References}

1 Kurtzman N. Chronic renal failure: metabolic and clinical consequences. Hospital Practice 1982 (August): 107-22.

2 Lazurus $M$. Dialytic therapy: principles and clinical guidelines. Hospital Practicc 1982 (October): 111-33.

3 Brenner B, Lazurus $M$. Chronic renal failure: pathophysiological and clinical considerations. Harrison's Principles of Internal Medicine, 10th Edition, p. 1612-27.

4 Grodstein, G, Harrison A, Roberts $C$ et al. Impaired gastric emptying in haemodialysis patients (abstr). Annual Meeting Am Soc Nephrol 1979; p. $182 \mathrm{~A}$.

5 Kuruvila C, Schrier R. Chronic renal failure. Intemational Anesthesiology Clinics 1984; 22 (1): 101-17

6 Opelz G. Terasaki PI. Dominant effects of transfusions on kidney graft survival. Transplantation 1980; 29: 153-8.

7 Morris $P J$. Blood transfusions and transplantation: transplantation 1978; 26: 276.

8 Van Rood J. Blood transfusion and transplantation. Transplantation 1978; 26: 275 .

9 Tasker PRW, MacGregor GA, DeWardener HE. Prophylactic use of intravenous saline in patients with chronic renal failure undergoing major surgery. Lancet 1974; 2: 911.

10 Rabiner $S F$, Drake $R F$. Platelet function as an indicator of adequate dialysis. Kidncy Int 1975; 7: $S 144$.

11 Stewart JH, Castaldi PA. Uraemic bleeding: a reversible platelet defect corrected by dialysis. QJ Med 1967; 143: 409.

12 Hampers $C L$, Blaufox $M D$, Merrill JP. Anticoagulation rebound after haemodialysis. N Eng J Med 1966; 275: 776.

13 Bastron RD, Deutsch $S$. Anaesthesia for the functionally anephric patient. Anaesthesia and the Kidney - The Scientific Basis of Clinical Anaesthesia, New York: Grune \& Stratton, 1976; p. 65

14 Ghanheim $M M$, Long JP. The interaction between magnesium and other neuromuscular blocking agents. Anesthesiology 1970; 32: 23.

15 Kraynach BJ. Calcium channel blocking agents: side effects and drug interaction. Annual Refresher Course Lectures, Chap. 238, 1983; American Society of Anesthesiologists.

16 Reves JG, Kissin I, Lell WA el al. Calcium entry 
blockers: uses and implications for anaesthesiologists. Ancsthcsiology 1982; 57: 504-18.

17 Breimer $D D$, Jochemsen $R$, Von Albert $H H$. Pharmacokinetics of benzodiazepenes. Drug Research 1980; 30: 875-81.

18 Husemeyer $R P$ et al. Cimetidine as a single oral dose for prophylaxis against Mendelson's syndrome Anaesthesia 1978; 33, 775-8.

19 Manchikanti $L$ et al. Cimetidine and related drugs in anesthesia. Anesth Analg 1982; 61: 595-608.

20 Carlier M. Squifflet JP et al. Maximal hydration during anaesthesia increases pulmonary artery pressure and improves function of human renal transplants. Transplantation 1982; 34: 201-4.

21 Wyant $G M$. The anaesthetists looks at tissue transplantation: three years experience with kidney transplants. Can Anaesth Soc J 1967; 14: 255.

22 Vandam LD, Harrison JH, Murray JE et al. Anaesthesia aspects of renal homotransplantation in man. Anesthesiology 1962; 23: 783.

23 Linke $C L$, Merin $R G$. A regional angesthetic approach for renal transplantation. Anesth Analg 1976; 55: 69-73.

24 Bromage PR. Gertel $M$. Brachial plexus anesthesia in chronic renal failure. Anesthesiology 1972; 36: 488-93.

25 Maddern PJ. Anaesthesia for the patient with inpaired renal function. Anaesth Intensive Care 1983; 11: 321-8.

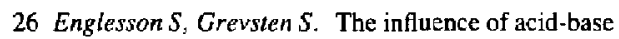
changes on central venous sytem toxicity of local anaesthetics. Acta Anaesthesiol Scand 1974; 18: 88-103.

27 Gould $D B$, Aldrete JA. Bupivicaine cardiotoxicity in a patient with renal failure. Act Anaesthesiol Scand 1983; 27: 18-21.

28 Ghoneim, Pandya $H$. Plasma protein binding of thiopental inpatients with impaired renal or hepatic function. Anesthesiology 1975; 42: 545.

29 Freeman $R B$, Sheff $M F$ et al. The blood-cerebrospinal fluid barrier in uraemia. Ann Int Med 1962; 56: 233.

30 Gorman HM, Graythorne NWB. The effect of a new neuroleptic analgesic agent (Innovar) on renal function in mant. Acta Anaesthesiol Scand Suppl 1966; 24 (10): 111.

31 Morgan $M$, Lumley $J$. Anaesthetic considerations in chronic renal failure. Anaesth Intensive Care 1975; 3: 218 .

32 Trudnawski RJ et al. Neuroleptanalgesia for patients with kidney malfunction. Anesth Analg 1971; 50: 679 .

33 Kono K, Philbin DM, Coggins CH et al. Renal function and stress response during halothane or fentanyl anaesthesia. Anesth Analg 1981; 60: 552-6.

34 Philbin DM, Coggins CH. Plasma and diuretic hormone levels in cardiac surgical patients during morphine and halothane anesthesia. Anesthesiology 1978; 49: 95.

35 Don $H F$, Dipeea RA, Taylor $P$. Narcotic analgesics in anuric patients. Anesthesiology 1975; 42: 745 .

36 Drayes $D E$. Active drug metabolites and renal failure. Am J Med 1977; 62: 486-9.

37 Day S. Plasma potassium changes following suxamethonium in normal patients and in patients with renal failure. Br J Anaesth 1976; 48: 1011-4.

38 Miller RD, Way WL, Hamilton WK et al. Succiny]choline induced hyperkalemia in patients with renal failure. Anesthesiolngy 1972; 36: 138-41.

39 Walion $J D$, Farman $J V$. Suxamethonium hyperkalaemia in uracmic ncuropathy. Anacsthesia 1973; 28: $66-8$.

40 Ryan DW. Preoperative serum cholinesterase concentration in chronic renal failure. Br J Anaesth 1977; 49: 945-9.

41 McLeod K, Watson MJ, Rawlins MD. Pharmacokinetics of pancuronium in patients with normal and impaired renal function. Br J Anaesth 1976; 48 : $341-5$.

42 Bowman WG. Neuromuscular blocking agents. Pharmacology of neuromuscular function. John Wright, Bristol 1980; 71-121.

43 Miller RD et al. Enhancement of d-tubocurarine neuromuscular blockade by diuretics in man. Anesthesiology 1976; 45: 442-5.

44 Cohen EN, Brewer HW, Smith D. The metabolism and elimination of d-tubocurarine $-\mathrm{H}^{3}$. Anesthesiology 1967; 28: 309-17.

45 Meistelman $C$, Lienhart $A$, Leveque $C$ et al. Pharmacology of vecuronium in patients with end stage renal failure. Anesthesiology 1983; 59: 293.

46 Hunter $J M$, Jones RS, Utting $J E$. Use of the muscle relaxant altracurium in ancphric patients. Prelim Comm J Royal Society of Medicine 1982; 75; 33640.

47 Fahey MR et al. Pharmacokinetics of atracurium in normal and renal failure patients. Anesthesiology 1983; 59: A263. 
48 Cousins MJ, Mazze RI. Methoxyflurane nephrotoxicity. A study of dose response in man. JAMA 1973; 225: 1611-6.

49 Cousins MJ, Greenstein LR, Hitt BA. Metabolism and renal effects of enflurane in man. Anesthesiology 1976; 44: 44.

50 Mazze RI, Stevenpiper JS. Renal effects of enflurane and halothane in patients with abnormal renal function. Anesthesiology 1984; 60: 161-3.

51 Harnett MN, Lane W, Bennett WM. Non-oliguric renal failure and enflurane. Ann Int Med 1974; 81: 560 .

52 Lachning R, Mazze RI. Possible mephrotoxicity from enflurane in patient with severe renal disease. Anesthesiology, 1974, 40: 203-5.

53 Eichorn $/ H$. Hedley-Whyte $J$ et al. Renal failure following enflurane anesthesia. Anesthesiology, 1976; 45: 557-60.

54 Mazze RI, Cousins MJ, Barr GA. Renal effects and metabolism of isoflurane in man. Anesthesiology, 1974; 40: 536-42.

55 Berry AJ. Respiratory support and renal function. Anesthesiology 1981; 55: 655-67.

56 Stanski $D R$, Miller $R D$, Sheiner $L B$. Renal function and the pharmacokinetics of neostigmine in anesthetized man. Anesthesiology 1979; 51: 222-6.

57 Cronnelly $R$, Stanski $D R$, Miller RD, Sheiner $L B$. Pyridostigmine kinetics with and without renal function. Clin Pharmacol Ther 1980; 28: 78-81.

58 Miller RD, Cullen DJ. Renal failure and postoperative failure: recurarization $\mathrm{Br} J$ Anacsth 1976; 48 : 253-6.

59 Miller RD. Reversal of neuromuscular blockade. 1978 Annual Refresher Course Lectures. Lecture 216. American Society of Anesthesiologists. 